\title{
Correction to: Prevalence of hypertension and diabetes after exposure to extracorporeal shock-wave lithotripsy in patients with renal calculi: a retrospective non-randomized data analysis
}

\author{
Christian Daniel Fankhauser ${ }^{1}$ - Nilufar Mohebbi ${ }^{2}$. Josias Grogg ${ }^{1}$ - Alexander Holenstein ${ }^{1}$ - Qing Zhong ${ }^{3,4}$. \\ Thomas Hermanns ${ }^{1} \cdot$ Tullio Sulser $^{1} \cdot$ Johann Steurer ${ }^{5}$. Cédric Poyet ${ }^{1}$
}

Published online: 19 July 2018

(c) The Author(s) 2018

\section{Correction to: \\ International Urology and Nephrology \\ (2018) 50:1227-1233 \\ https://doi.org/10.1007/s11255-018-1857-2}

The ninth author name was incorrectly published in the original publication. The correct name should read as 'Cédric Poyet'.
Open Access This article is distributed under the terms of the Creative Commons Attribution 4.0 International License (http://creativeco mmons.org/licenses/by/4.0/), which permits unrestricted use, distribution, and reproduction in any medium, provided you give appropriate credit to the original author(s) and the source, provide a link to the Creative Commons license, and indicate if changes were made.

The original article can be found online at https://doi.org/10.1007/ s11255-018-1857-2.

Christian Daniel Fankhauser

christian.fankhauser@usz.ch

1 Department of Urology, University Hospital Zurich, University of Zurich, Zurich, Switzerland

2 Division of Nephrology, University Hospital Zurich, University of Zurich, Zurich, Switzerland

3 Department of Pathology of Molecular Pathology, University Hospital Zurich, University of Zurich, Zurich, Switzerland

4 Cancer Data Science Group, Children's Medical Research Institute, University of Sydney, Sydney, NSW, Australia

5 Horten Centre for Patient Oriented Research and Knowledge Transfer, University Hospital Zurich, University of Zurich, Zurich, Switzerland 